\title{
A Novel Reconfigurable Metasurface with Coincident and Ultra-Wideband LTL and LTC Polarization Conversion Functions
}

\author{
Zexu GUO, Xiangyu CAO, Jun GAO, Huicun YU, Jiangfeng HAN, Huanhuan YANG, Jianghao TIAN
}

Information and Navigation College of Air Force Engineering University, Xi'an, Shaanxi, 710077, China

418604809@qq.com

Submitted July 6, 2019 / Accepted August 16, 2019

\begin{abstract}
In this paper, a novel reconfigurable metasurface (NRM) with coincident and ultra-wideband linear-to-linear (LTL) and linear-to-circular (LTC) polarization conversion functions is proposed. The unit of the proposed NRM consists of superstrate, air layer, metal patch, substrate and metal ground successively. By loading micro-electromechanical system (MEMS) between two adjacent metallic via, the proposed NRM can realize LTL and LTC polarization conversion efficiently. Numerical simulation results reveal that the bandwidth of LTL and LTC polarization conversion are coincident from $5.7 \mathrm{GHz}$ to $23.5 \mathrm{GHz}$ (fractional bandwidth of $122 \%$ ). It is worth mentioning that few metasurface in existing literature can achieve highly coincident and ultra-wideband LTL and LTC polarization conversion functions. Finally, measurement results are in accordance with simulation results.
\end{abstract}

\section{Keywords}

Reconfigurable, ultra-wideband, polarization conversion

\section{Introduction}

Metasurface, as an artificially engineered material, had attracted the attention of many scholars. Metasurface possessed many potential applications [1-4], which provide an alternative scheme to manipulate the electromagnetic waves by arranging the artificial structures [5-8].

In recent years, with application of polarization in information transmission, the polarization controllable devices are more and more essential. Polarization converters can manipulate electromagnetic (EM) waves artificially, which have been applied in many areas [9-15].

Polarization converters based on metasurface have been designed due to their many unique characteristics. The implementation of the existing reconfigurable polarization converters can be divided into two methods. One method is to change the dielectric materials [16-21], the other is to change effective metal structure by using electronic devices [22-33]. The former method uses materials such as liquid crystals [16], graphene [17], [18], metal fluid [19], [20], vanadium oxide $\left(\mathrm{VO}_{2}\right)$ film [21] to replace the traditional metal and dielectric materials, this method has the characteristics of high frequency band and difficult fabrication. In contrast, the latter method uses PIN diodes [22-27], varactor diode [28], [29] and MEMS switch [30], [31] which have the characteristics of easy fabrication, good electronic control performance, flexible structure and high efficiency. For example, an active metasurface has been proposed in [29], which could convert linear polarization waves into orthogonal polarization waves and circular polarization waves. In our previous work [30], the proposed reconfigurable polarization converter could reflect the x-polarization waves to y-polarization waves and circular polarization waves from $8.07 \mathrm{GHz}$ to $10.77 \mathrm{GHz}$. Performance comparison of reconfigurable polarization converters is given in Tab. 1.

Although there are many researches about the reconfigurable polarization conversion based on metasurface, wider bandwidths still need further study. To date, in existing reconfigurable polarization converters, the common bandwidth of the two different polarization conversion states is narrow, which limits their application.

In this paper, a NRM with coincident and ultra-wideband LTL and LTC polarization conversion functions is proposed. Two kinds of current loops are realized by loading switching device in the strong induced current part. Compared with the previous polarization converter, the proposed NRM only loads one MEMS of each unit but can achieve highly coincident and ultra-wideband LTL and LTC polarization conversion functions. The NRM has advantages of multi-polarization, ultra-wideband, fewer electronic devices and high efficiency.

\begin{tabular}{|c|c|c|c|}
\hline & $\begin{array}{c}\text { LTL } \\
\text { polarization } \\
\text { conversion } \\
(\mathrm{GHz})\end{array}$ & $\begin{array}{c}\text { LTC } \\
\text { polarization } \\
\text { conversion } \\
(\mathrm{GHz})\end{array}$ & $\begin{array}{c}\text { The } \\
\text { coincident } \\
\text { bandwidth } \\
(\mathrm{GHz})\end{array}$ \\
\hline$[29]$ & $6.5-19.9$ & $7.6-23.6$ & $7.6-19.9$ \\
\hline$[30]$ & $7.93-12.42$ & $8.07-10.77$ & $8.07-10.77$ \\
\hline This paper & $5.6-23.5$ & $5.7-23.8$ & $5.7-23.5$ \\
\hline
\end{tabular}

Tab. 1. Performance comparison table of reconfigurable polarization converter. 


\section{Design and Simulation}

Based on our previous studies [26], [30], we found that by loading switching device in the strong induced current part, two kinds of current loops and polarization reconfiguration can be realized. The proposed NRM is based on an ultra-wideband single polarization converter [15]. The MEMS switch is loaded between two adjacent metallic vias where the induced current is strong, multipolarization can be realized by controlling MEMS switch.

The unit of the NRM consists of superstrate, air layer, metal patch, substrate and metal ground successively, as shown in Fig. 1(a). The same material $\left(\varepsilon_{\mathrm{r}}=2.2\right.$ and $\tan \delta=0.0009)$ is used in substrate and the superstrate. The actual conditions for loading MEMS switch should be satisfied. The distance $g_{2}$ should be greater than $1 \mathrm{~mm}$, and the air layer $h_{2}$ should be at least $1 \mathrm{~mm}$, as shown in Fig. 1(b). At the same time, the bandwidth of LTL and LTC polarization conversion should be coincident as far as possible. The unit structural parameters are given in Tab. 2 . Radant RF MEMS switch RMSW200HP is selected as the intended object. In order to facilitate simulation, it is simplified into a metal bridge structure. According to the literature [30], [34], this method is feasible. When the switch is on, MEMS switch is simplified into a metal bridge, as shown in Fig. 1(b). When the switch is off, the MEMS switch is simplified as nothing, as shown in Fig. 1(c). The yellow parts represent copper and blue parts represent substrate.

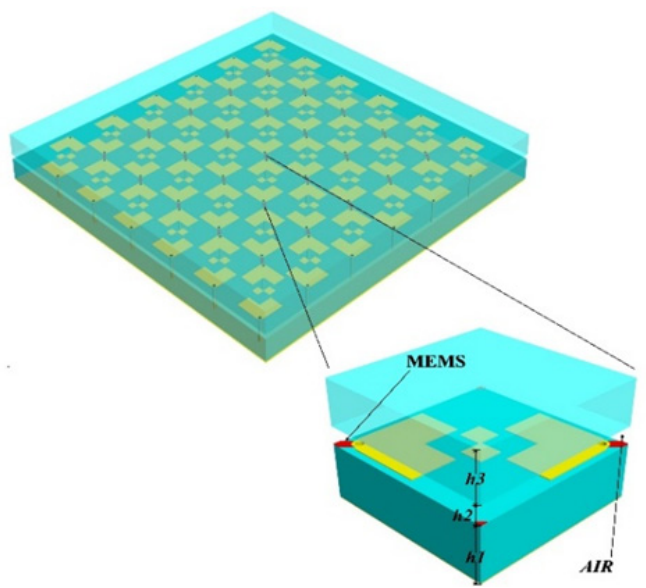

(a)

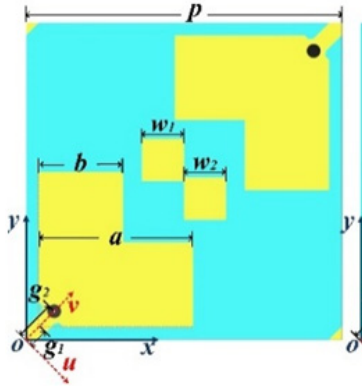

(b)

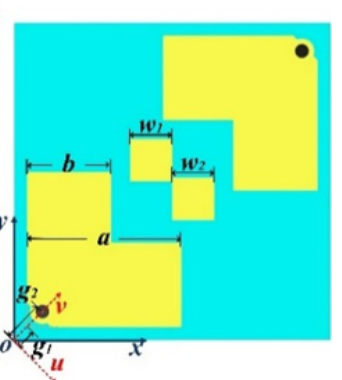

(c)
Fig. 1. Schematic of the designed NRM: (a) Perspective view; (b) when the MEMS switch is on; (c) when the MEMS switch is off.

\begin{tabular}{|c|c|c|c|}
\hline parameter & Size $(\mathrm{mm})$ & parameter & Size $(\mathrm{mm})$ \\
\hline$a$ & 4.4 & $w_{1}$ & 1.1 \\
\hline$b$ & 2.4 & $w_{2}$ & 1.2 \\
\hline$h_{1}$ & 3 & $g_{1}$ & 0.51 \\
\hline$h_{2}$ & 1 & $g_{2}$ & 1.13 \\
\hline$h_{3}$ & 2.5 & $p$ & 9 \\
\hline
\end{tabular}

Tab. 2. The unit structural parameters of the NRM (mm).

Electromagnetic simulation software Ansoft HFSS is used to simulate the proposed NRM. We set the x-polarized waves as the incident waves. We use $R_{x x}=e_{r x} / e_{i x}$, $R_{y x}=e_{r y} / e_{i x}, \varphi_{x x}=\delta_{r x}-\delta_{i x}$ and $\varphi_{y x}=\delta_{r y}-\delta_{i x}$ to represent the coefficient of co-polarized reflections, the coefficient of cross-polarized reflections, the phases of co-polarized reflections and the phases of cross-polarized reflections, respectively.

When the MEMS switch is on, the amplitudes of $R_{y x}$ is much larger than $R_{x x}$ in the extremely wide frequency band, as shown in Fig. 2(a). $R_{y x}$ is very close to 1 at frequencies of $6.2 \mathrm{GHz}, 8.8 \mathrm{GHz}, 12.4 \mathrm{GHz}, 16.2 \mathrm{GHz}, 21.5 \mathrm{GHz}$ and $23.5 \mathrm{GHz}$, indicating that $\mathrm{x}$-polarized incident waves are converted into y-polarized reflection waves efficiently. When the MEMS switch is off, the amplitudes of x-polarized reflection waves and y-polarized reflection waves are very close, as shown in Fig. 2(b).

Black and blue lines represent reflected phase, red lines represent reflected phase difference in Fig. 2(c) and 2(d). The reflected phase difference between x-polarized incident waves and $\mathrm{y}$-polarized reflection waves keeps approximately $\Delta \varphi=90^{\circ} \pm 2 k \times 180^{\circ}$ from $5 \mathrm{GHz}$ to $23 \mathrm{GHz}$, as shown in Fig. 2(c) and 2(d).

Therefore, when the switch is off, linearly polarized incident waves can be rotated into circularly polarized reflection waves. The PCR (Polarization Conversion Ratio) is calculated as (1) and Axis Ratio (AR) is defined as (2) [35].

$$
\begin{gathered}
P C R=R_{y x}{ }^{2} /\left(R_{x x}{ }^{2}+R_{y x}{ }^{2}\right), \\
A R=\mid 20 \log _{10} \tan \left[0.5 \arcsin \left(\frac{2 R_{x x} R_{y x}}{R_{x x}{ }^{2}+R_{y x}^{2}} \sin \Delta \varphi\right)\right] .
\end{gathered}
$$

The PCR of the NRM is shown in Fig. 3(a). When the switch is on, the PCR is higher than $90 \%$ ranging from $5.7 \mathrm{GHz}$ to $23.8 \mathrm{GHz}$, and the fractional bandwidth is $122.7 \%$. Hence, $\mathrm{x}$-polarized incident waves are converted into their orthogonal polarized reflection waves. When the MEMS switch is off, the AR of circularly polarized reflection wave is shown in Fig. $3(\mathrm{~b})$. The $3 \mathrm{~dB}$ bandwidth is $5.6 \mathrm{GHz}$ to $23.5 \mathrm{GHz}$, and the fractional bandwidth is $123 \%$.

According to the above analysis, the bandwidth of the two different polarization states is highly coincident. Therefore, the proposed NRM could achieve LTL and LTC 
polarization conversion from $5.7 \mathrm{GHz}$ to $23.5 \mathrm{GHz}$, the fractional bandwidth is $122 \%$.

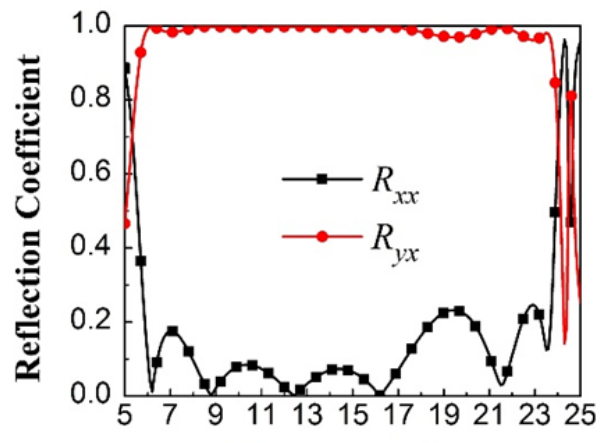

Frequency/GHz

(a)

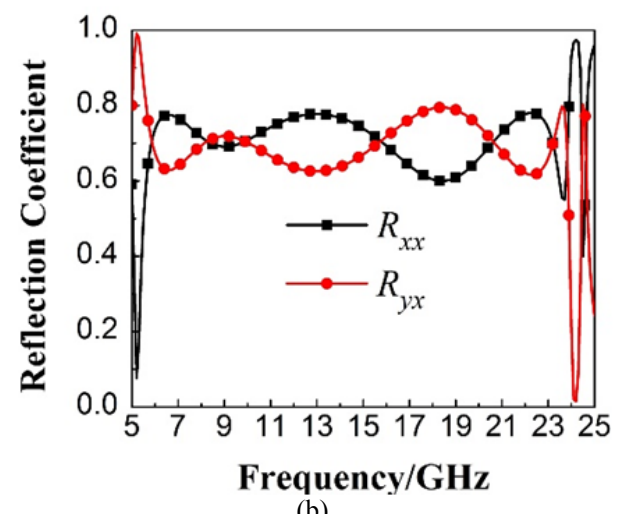

(b)

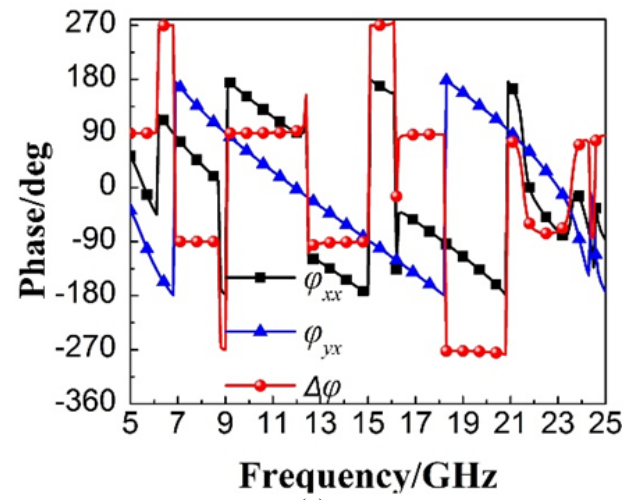

(c)

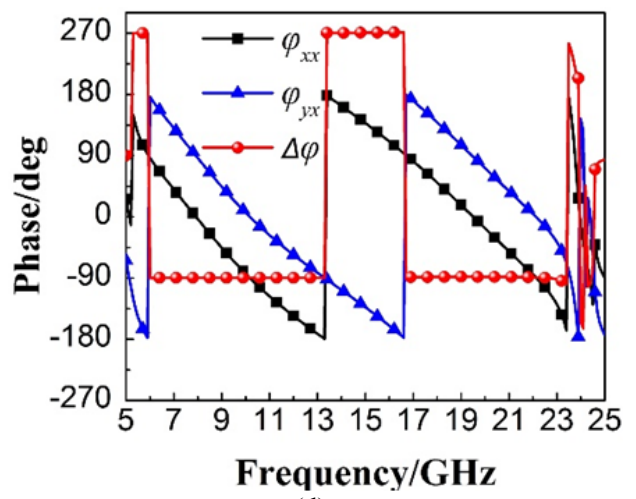

(d)

Fig. 2. When the switch is on, reflection coefficient is shown in (a) and phase is shown in (c). When the switch is off, reflection coefficient is shown in (b) and phase is shown in (d).

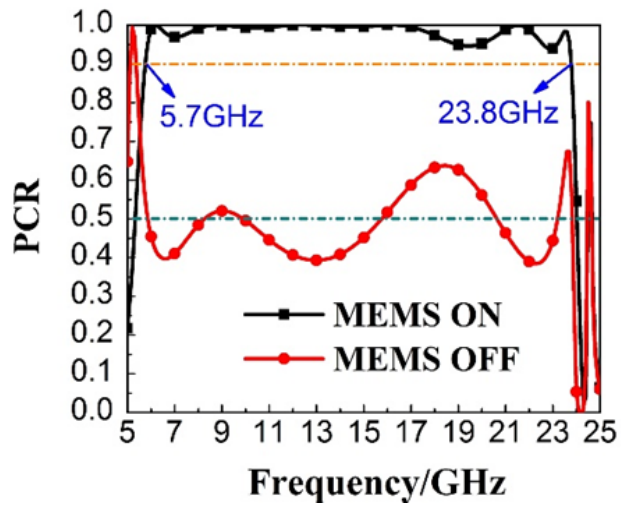

(a)

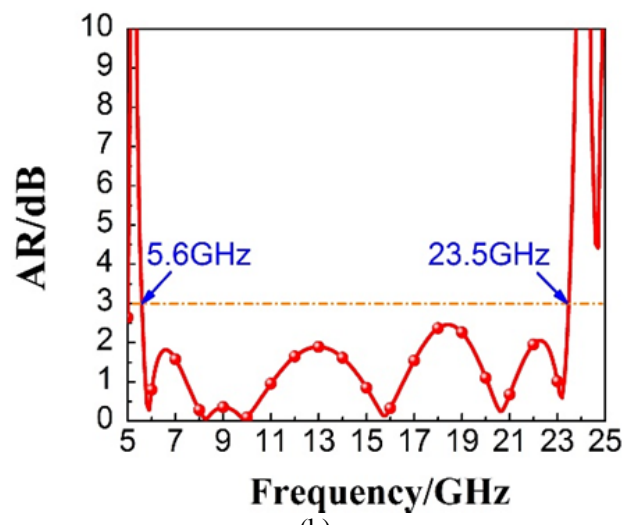

(b)

Fig. 3. PCR of reflected waves is shown in (a) when the MEMS switch is off, AR of circularly polarized reflected waves is shown in (b).

\section{Results and Discussion}

In order to analyze the physical mechanisms of the designed NRM better, the electric field is decomposed into $\mathrm{u}-\mathrm{v}$ coordinate system, as shown in Fig. 1(b) and 1(c). We define $R_{u u}$ to represent the reflection coefficient of u-to-upolarization, $R_{v u}$ to represent the reflection coefficient of v-to-u-polarization, $R_{u v}$ to represent the reflection coefficient of $\mathrm{u}$-to-v-polarization and $R_{v v}$ to represent the reflection coefficient of v-to-v-polarization, respectively [30].

There are the simulated reflection coefficients, as shown in Fig. 4(a) and 4(b). It reflects that $R_{u u} \approx R_{v v} \approx 1$, $R_{u v} \approx R_{v u} \approx 0$, indicating all the reflected waves are co-polarized reflections. Furthermore, the co-polarized reflection coefficient is very close to 1 , which indicates that the proposed NRM is highly efficient in the polarization conversion process. When the frequency is higher than $23 \mathrm{GHz}$, cross-polarized components appear in the reflected waves.

We define $\varphi_{u u}$ to represent the reflection phases of $\mathrm{u}$ to-u, and $\varphi_{v v}$ to denote v-to-v the reflection phases of polarization conversions. $\Delta \varphi^{\prime}$ denotes the corresponding phase difference between $\varphi_{u u}$ and $\varphi_{v v}$. The simulated reflection phase is exhibited in Fig. 4(c) and 4(d). When the MEMS switch is on, $\Delta \varphi^{\prime}$ fluctuates around $\pm 180^{\circ}$, as shown in Fig. 4(c). At this moment, u-polarization and v-polarization compose y-polarized reflected waves, indicating that 
the proposed NRM rotates linearly polarized incident wave to its orthogonal polarization.

In order to understand the circular polarization characteristics better, we analyze the elliptical polarization. $\chi$ represents the elliptical angle. $a$ is the long axis and $b$ is the short axis of an ellipse:

$$
\begin{gathered}
\chi=\arctan (b / a)(-\pi / 4 \leq \chi \leq \pi / 4), \\
\sin (2 \chi)=\frac{2 e_{r u} e_{r v}}{{e_{r u}{ }^{2}+e_{r v}{ }^{2}}^{2}} \sin \Delta \varphi^{\prime} .
\end{gathered}
$$

According to previous analysis, the reflected amplitudes of $u$ and $v$ axis are equal. Since the coordinate axis of

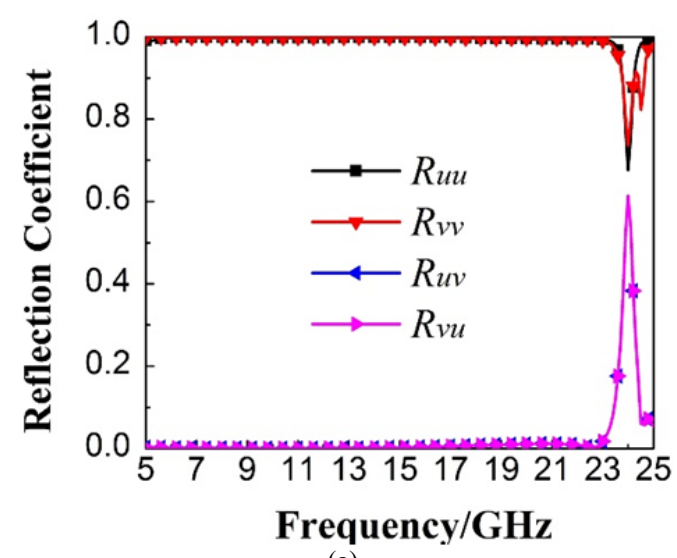

(a)

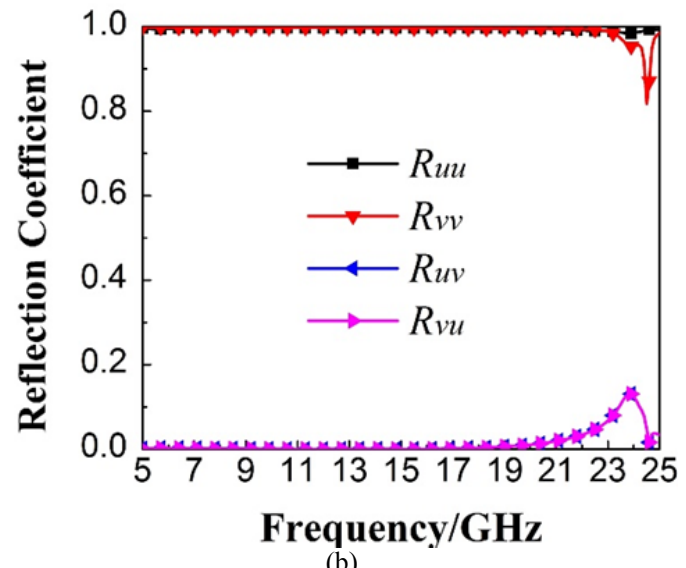

(b)

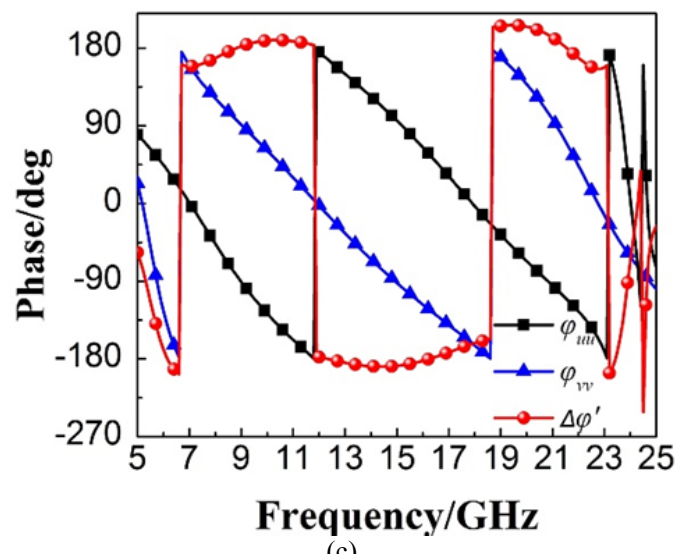

(c)

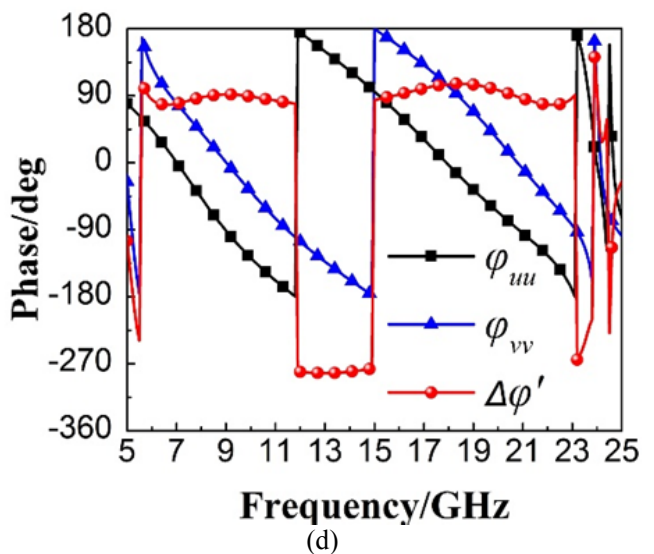

Fig. 4. The reflection coefficients in $\mathrm{u}-\mathrm{v}$ coordinate system. When the switch is on, reflection coefficient is shown in (a) and phase is shown in (c). When the switch is off, reflection coefficient is shown in (b) and phase is shown in (d).

$\mathrm{u}$ and $\mathrm{v}$ are orthogonal, the AR has been obtained as the following formula.

$$
A R=\left|20 \log _{10}(b / a)\right|=\left|20 \log _{10} \tan \left(0.5 \Delta \varphi^{\prime}\right)\right| .
$$

If the reflected wave is circular polarization, the $A R$ must be less than $3 \mathrm{~dB}$ in engineering. According to (5), $\Delta \varphi^{\prime}$ should satisfy $\left[71^{\circ}+180^{\circ} \times k, 109^{\circ}+180^{\circ} \times k\right]$, in which $k$ is integer. When the switch is off, $\Delta \varphi^{\prime}$ fluctuates around $90^{\circ}$ and $-270^{\circ}$ from $5.6 \mathrm{GHz}$ to $23.5 \mathrm{GHz}$, as shown in Fig. 4(d). The reflected waves are circular polarization. And the result is in accordance with the result that is obtained under $\mathrm{x}-\mathrm{y}$ coordinates.

Compared with Fig. 4(c) and 4(d), the two curves of $\varphi_{u u}$ are almost the same, but the two curves of $\varphi_{v v}$ are obviously changed due to the on-off of the switch. It indicates that it has no impact on u-polarized waves regardless of whether the switch is on or off, while it has the impact on the v-polarized waves. Therefore, the induced current distribution based on v-polarized incident waves should be further analyzed.

Under v-polarized incidences, the induced current distributions at the resonant frequencies $(6.2 \mathrm{GHz}, 8.8 \mathrm{GHz}$, 12.4 GHz, $16.2 \mathrm{GHz}, 21.5 \mathrm{GHz}$ and $23.5 \mathrm{GHz}$ ) are drawn successively, as shown in Fig. 5(a) and Fig. 5(b). With the increase of frequency, it is obvious that the distribution of the induced current gradually flows through the metallic via and switch to a small square metal patch. In addition, when the switch is on, the induced current mainly distributes around the switch. When the switch is off, the induced current mainly distributes around the metallic via and the long side of L-shaped metal patch, indicating that the onoff of the switch can change the distribution of the induced current significantly.

The intensity of resonance is stronger, indicating that the higher PCR will be realized. As shown in Fig. 5(a) and Fig. 5(b), the amplitude of "switch-on" induced current is about twice as much as the "switch-off" induced current, which corresponds to the PCR reduces half of switch on after 


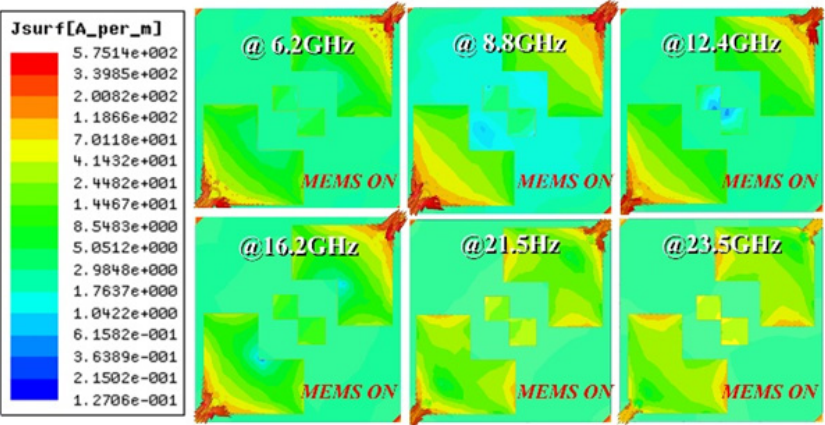

(a)

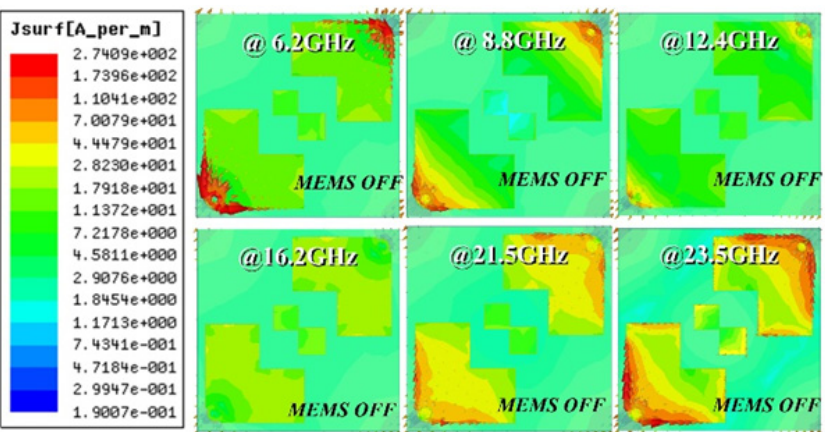

(b)

Fig. 5. The top view of induced current distributions under v-polarized incident wave: (a) When the MEMS switch is on, and (b) when the MEMS switch is off.

switching off, as shown in Fig. 3(a). Therefore, the proposed NRM can achieve LTL and LTC polarization conversion in one metasurface simultaneously, which is consistent with the original conclusion.

\section{Experimental Results}

To further validate this design, the NRM device was fabricated. As shown in Fig. 6(a), the practical sample is composed of $(30 \times 30)$ units, and the overall structural size of the sample is $270 \mathrm{~mm} \times 270 \mathrm{~mm} \times 6.5 \mathrm{~mm}$. The air layer is supported by $1 \mathrm{~cm}$ thick nylon gasket at four corners of the sample. To simplify manufacturing, a metallic connection bridge is used to take the place of the MEMS switch in this experiment. According to [30], this method is reliable. Space wave method [36] was used to test the sample in the microwave anechoic chamber, as shown in Fig. 6(b). Above all, we define the horizontal orientation as the $\mathrm{x}$ polarization, and the vertical orientation as $y$-polarization. The transmitting and receiving horn antenna are placed in X-polarization. $R_{x x}$ and $\varphi_{x x}$ were measured respectively. Next, the transmitting horn antenna is placed in y-polarization, and $R_{x y}$ and $\varphi_{x y}$ were measured respectively.

Based on the measured results, we can calculate the PCR and AR, according to (1) and (2). Owing to the limitation of experimental conditions, the proposed NRM is measured less than $18 \mathrm{GHz}$. The comparison of measurement and simulation is shown in Fig. 6(c) and (d). Although limited by the frequency of measurement, the trend of simulated and measured curves is roughly same. From the experimental results, we can find that the proposed NMR

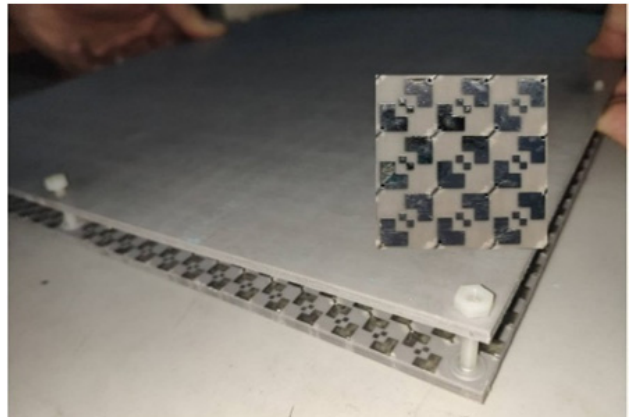

(a)

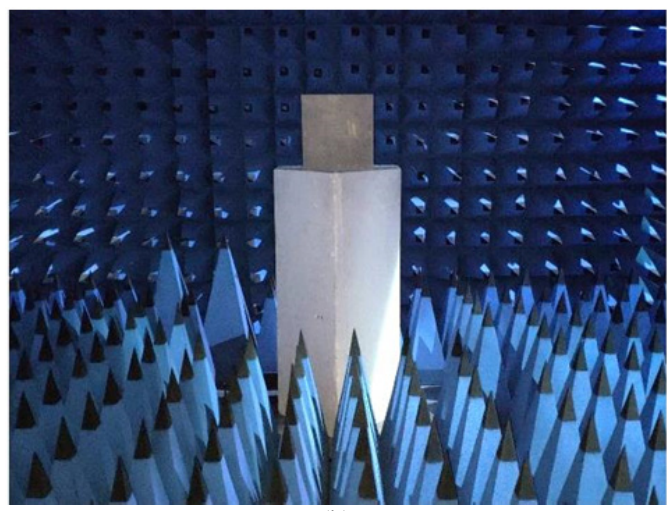

(b)

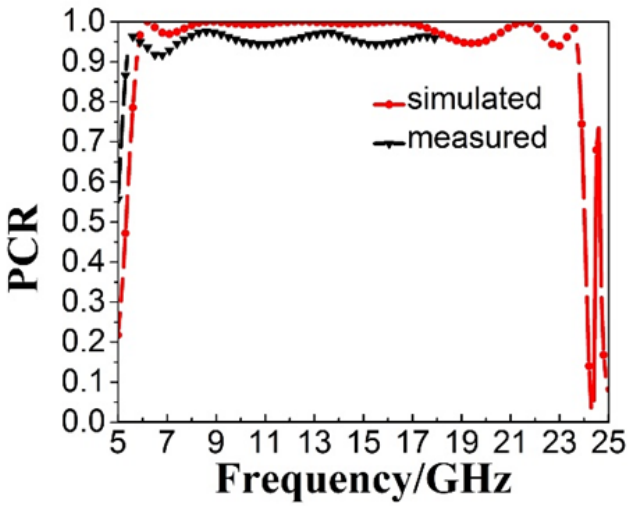

(c)

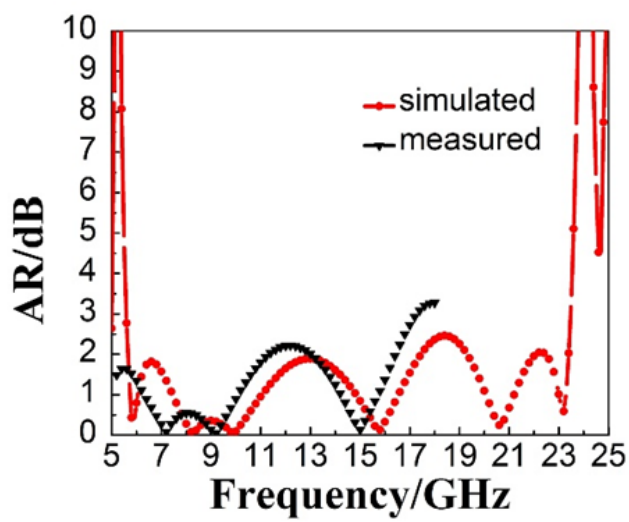

(d)

Fig. 6. (a) NRM device. (b) Microwave anechoic chamber. (c) The simulated PCR and measured PCR. (d) AR of simulated and measured results.

can achieve LTC polarization conversion efficiently, and LTL polarization conversion with the PCR is greater than $90 \%$. Because the experimental measurement is not 
an ideal state and because of the accuracy of fabrication, there is discrepancy among measurement and simulation results. Hence, the validity of the simulation is verified by the experimental measurements.

\section{Conclusion}

We proposed a novel reconfigurable metasurface. When the switch is on, the proposed polarization converter could achieve LTL polarization conversion ranging from $5.6 \mathrm{GHz}$ to $23.5 \mathrm{GHz}$ with the polarization conversion ratio over $90 \%$. When the switch is off, it could achieve LTC polarization conversion ranging from $5.7 \mathrm{GHz}$ to $23.8 \mathrm{GHz}$ with the AR less than $3 \mathrm{~dB}$. To further validate the mechanism, the electromagnetic wave was decomposed into $\mathrm{u}-\mathrm{v}$ direction and the distribution of induced current was analyzed, which can testify the reconfigurable feature of it.

It is worth mentioning that the bandwidth of LTL and LTC polarization conversion are highly coincident from $5.7 \mathrm{GHz}$ to $23.5 \mathrm{GHz}$ (fractional bandwidth of $122 \%$ ). The measured results are in accordance with simulated results. The designed NRM has potential application in the dynamic modulation of antenna polarization.

\section{Acknowledgments}

National Natural Science Foundation of China under grant (Nos. 61671414, 61801508, 61701523 and 61471389); Natural Science Basic Research Program of Shaanxi Province, China (Grant Nos. 2019JQ-103, 2018JM6040 and 2017JM6025); Postdoctoral Innovative Talents Support Program of China (BX20180375) and China Postdoctoral Science Foundation (2019M650098).

\section{References}

[1] ZHU, X. W., GAO, J., CAO, X. Y., et al. A novel low-RCS and wideband circularly polarized patch array based on metasurface. Radioengineering, 2019, vol. 28, no. 1, p. 99-107. DOI: 10.13164/re.2019.0099

[2] SCHURIG, D., MOCK, J. J., JUSTICE, B. J., et al. Metamaterial electromagnetic cloak at microwave frequencies. Science, 2009, vol. 314 , no. 5801, p. 977-980. DOI: $10.1126 /$ science. 1133628

[3] NARIMANOV, E. E., KILDISHEV, A. V. Optical black hole: broadband omnidirectional light absorber. Applied Physics Letters, 2009, vol. 95, no. 4, p. 1-3. DOI: 10.1063/1.3184594

[4] LI, S., GAO, J., CAO, X., et al. Multiband and broadband polarization-insensitive perfect absorber devices based on a tunable and thin double split-ring metamaterial. Optics Express, 2015, vol. 23, no. 3, p. 3523-3533. DOI: 10.1364/oe.23.003523

[5] SONG, X., HUANG, L., SUN, L., et al. Near-field plasmonic beam engineering with complex amplitude modulation based on metasurface. Applied Physics Letters, 2018, vol. 112, no. 7, p. 1-5. DOI: $10.1063 / 1.5013327$

[6] ZHANG, C., CAO, X. Y., GAO, J., et al. Shared aperture metasurface for bi-functions: radiation and low backward scattering performance. IEEE Access, 2019, vol. 7, no. 1, p. 56547-56555. DOI: 10.1109/ACCESS.2019.2913726

[7] ZHANG, L., MEI, S., HUANG, K., et al. Advances in full control of electromagnetic waves with metasurfaces. Advanced Optical Materials, 2016, vol. 4, no. 6, p. 818-833. DOI: 10.1002/adom.201500690

[8] CAI, T., WANG, G. M., TANG, S. W., et al. High-efficiency and full-space manipulation of electromagnetic wave fronts with metasurfaces. Physical Review Applied, 2017, vol. 8, no. 3, p. 1-11. DOI: 10.1103/PhysRevApplied.8.034033

[9] ZHANG, L., ZHOU, P., LU, H. H., et al. Ultra-thin reflective metamaterial polarization rotator based on multiple plasmon resonances. IEEE Antennas and Wireless Propagation Letters, 2015, vol. 14, p. 1157-1160. DOI: 10.1109/lawp.2015.2393376

[10] PU, M., CHEN, P., WANG, Y., et al. Anisotropic meta-mirror for achromatic electromagnetic polarization manipulation. Applied Physics Letters, 2013, vol. 102, p. 1-4. DOI: 10.1063/1.4799162

[11] ZHOU, G., TAO, X., SHEN, Z., et al. Designing perfect linear polarization converters using perfect electric and magnetic conducting surfaces. Scientific Reports, 2016, vol. 6, no. 1, p. 1-10. DOI: $10.1038 /$ srep38925

[12] LAWRENCE, M., XU, N. N., ZHANG, X. Q., et al. Manifestation of symmetry breaking in polarization space with terahertz metasurfaces. Physical Review Letters, 2012, vol. 113 p. 1-5. DOI: 10.1103/PhysRevLett.113.093901

[13] AKGOL, O., UNAL, E., ALTINTAS, O., et al. Design of metasurface polarization converter from linearly polarized signal to circularly polarized signal. Optik, 2018, vol. 161, p. 12-19. DOI 10.1016/j.ijleo.2018.02.028

[14] JIANG, Y., WANG, L., WANG, J., et al. Ultra-wideband highefficiency reflective linear-to-circular polarization converter based on metasurface at terahertz frequencies. Optics Express, 2017, vol. 25 , no. 22 , p. 1-8. DOI: 10.1364/OE.25.027616

[15] JIA, Y. T., LIU, Y., ZHANG, W. B., et al. Ultra-wideband metasurface with linear-to-circular polarization conversion of an electromagnetic wave. Optical Materials Express, 2018, vol. 8, no. 3, p. 597-604. DOI: $10.1364 /$ ome.8.000597

[16] DOUMANIS, E., GOUSSETIS, G., DICKIE, R., et al. Electronically reconfigurable liquid crystal based mm-wave polarization converter. IEEE Transactions on Antennas and Propagation, 2014, vol. 62, no. 4, p. 2302-2307. DOI: 10.1109/TAP.2014.2302844

[17] CHENG, H., CHEN, S., YU, P., et al. Dynamically tunable broadband mid-infrared cross polarization converter based on graphene metamaterial. Applied Physics Letters, 2013, vol. 103, no. 22 , p. $1-4$. DOI: $10.1063 / 1.4833757$

[18] OWITI, E. O., YANG, H., LIU, P., et al. Polarization converter with controllable birefringence based on hybrid all-dielectricgraphene metasurface. Nanoscale Research Letters, 2018, vol. 13, p. 1-9. DOI: 10.1186/s11671-017-2413-1

[19] MA, R., LI, X., DONG, X., et al. Magnetic field modulating inline fiber polarization modulator based on microfiber and magnetic fluid. Applied Physics Letters, 2017, vol. 111, no. 9, p. 1-4. DOI: $10.1063 / 1.4985114$

[20] RUFF, A., LI, Z., LOIDL, A., et al. Frequency dependent polarisation switching in h-ErMnO 3 . Applied Physics Letters, 2018, vol. 112 , no. 18 , p. 1-5. DOI: $10.1063 / 1.5026732$

[21] WANG, H., MELlan, T. A., GRAU-CRESPO, R., et al. Spin polarization, orbital occupation and band gap opening in vanadium dioxide: The effect of screened Hartree-Fock exchange. Chemical Physics Letters, 2014, vol. 608, p. 126-129. DOI: 10.1016/j.cplett.2014.05.070

[22] LI, W., GAO, S., CAI, Y., et al. Polarization-reconfigurable circularly polarized planar antenna using switchable polarizer. 
IEEE Transactions on Antennas and Propagation, 2017, vol. 65, no. 9, p. 4470-4477. DOI: 10.1109/TAP.2017.2730240

[23] ZHANG, M. T., GAO, S., JIAO, Y. C., et al. Design of novel reconfigurable reflect arrays with single-bit phase resolution for ku-band satellite antenna applications. IEEE Transactions on Antennas and Propagation, 2016, vol. 64, no. 5, p. 1634-1641. DOI: 10.1109 /TAP.2016.2535166

[24] TAO, Z., WAN, X., PAN, B. C., et al. Reconfigurable conversions of reflection, transmission, and polarization states using active metasurface. Applied Physics Letters, 2017, vol. 110, no. 12, p. 1-5. DOI: $10.1063 / 1.4979033$

[25] CUI, J., HUANG, C., PAN, W., et al. Dynamical manipulation of electromagnetic polarization using anisotropic meta-mirror Scientific Reports, 2016, vol. 6, p. 1-9. DOI: 10.1038/srep30771

[26] GAO, X., HAN, X., CAO, W. P., et al. Ultra-wideband and highefficiency linear polarization converter based on double $\mathrm{v}$-shaped metasurface. IEEE Transactions on Antennas and Propagation, 2015, vol. 63, no. 8, p. 3522-3530. DOI: 10.1109/TAP.2015.2434392

[27] FARZAMI, F., KHALEDIAN, S., SMIDA, B., et al. Reconfigurable linear/circular polarization rectangular waveguide filtenna. IEEE Transactions on Antennas and Propagation, 2018, vol. 66, no. 1, p. 9-15. DOI: 10.1109/TAP.2017.2767634

[28] ZHU, H. L., CHEUNG, S. W., CHUNG, K. L., et al. Linear-tocircular polarization conversion using metasurface. IEEE Transactions on Antennas and Propagation, 2013, vol. 61, no. 9, p. 4615-4623. DOI: 10.1109/TAP.2013.2267712

[29] TIAN, J. H., CAO, X. Y., GAO, J., et al. A reconfigurable ultrawideband polarization converter based on metasurface incorporated with PIN diodes. Journal of Applied Physics, 2019, vol. 125 , no. 13 , p. 1-9. DOI: 10.1063/1.5067383

[30] YU, H. C., CAO, X. Y., GAO, J., et al. Design of wideband and reconfigurable polarization converter using manipulable metasurface. Optical Materials Express, 2018, vol. 8, no. 11, p. 3373-3381. DOI: 10.1364/OME.8.003373

[31] MA, X., PU, M., LI, X., et al. A planar chiral meta-surface for optical vortex generation and focusing. Scientific Reports, 2015, vol. 5, p. 1-7. DOI: $10.1038 /$ srep10365

[32] LI, T., YANG, H., LI, Q., et al. Dual-polarised and ultra-thin broadband for both $\mathrm{P}$ and $\mathrm{L}$ bands applications. IET Microwaves Antennas and Propagation, 2019, vol. 13, no. 2, p. 185-189. DOI: 10.1049/iet-map.2018.5151

[33] ZHANG, M., ZHANG, W., LIU, A. Q., et al. Tunable polarization conversion and rotation based on a reconfigurable metasurface. Scientific Reports, 2017, vol. 7, p. 1-7. DOI: 10.1038/s41598-01711953-z

[34] CHEN, W., GAO, J., ZHANG, G., et al. A wideband coding reflective metasurface with multiple functionalities. Acta Physica Sinica, 2017, vol. 66, no. 6, p. 1-8. DOI: 10.7498/aps.66.064203 (in Chinese)

[35] ZHANG, W., LI, J. Y., XIE, J. A broadband circular polarizer based on cross-shaped composite frequency selective surfaces. IEEE Transactions on Antennas and Propagation, 2017, vol. 65, no. 10, p. 5623-5627. DOI: 10.1109/TAP.2017.2735459

[36] YANG, H., CAO, X., YANG, F., et al. A programmable metasurface with dynamic polarization, scattering and focusing control. Scientific Reports, 2016, vol. 6, p. 1-11. DOI: $10.1038 /$ srep35692

\section{About the Authors...}

Zexu GUO was born in Hubei province, China. He received his B.S. degree from Beijing Jiaotong University in 2018. He currently works towards his M.S. degree. In his research, he specializes in metamaterial, polarization converter design and RCS reduction techniques.

Xiangyu CAO received her M.S. degree from the Air Force Missile Institute in 1989. In the same year, she joined the Air Force Missile Institute. She received her Ph.D. degree in the Missile Institute of AFEU in 1999. From 1999 to 2002, she was engaged in postdoctoral research in Xidian University, China. She was a Senior Research Associate in the Department of Electronic Engineering, City University of Hong Kong from June 2002 to Dec. 2003. She is currently a professor and a senior member of IEEE. Her research interests include computational electromagnetic, electromagnetic metamaterials and their antenna applications.

Jun GAO received the M.S. and Ph.D. degrees from the Air Force Missile Institute in 1984 and 1987, respectively. He joined the Air Force Missile Institute in 1987 as an assistant teacher. He became an associate professor in 2000. He is currently a professor of the Information and Navigation College, Air Force Engineering University of CPLA. He has authored and coauthored more than 100 technical journal articles and conference papers, and holds one China soft patent. His research interests include smart antennas, electromagnetic metamaterial and their antenna applications.

Huicun YU received the B.S. and M.S. degree from the Information and Navigation Institute, Air Force Engineering University of CPLA, China, in 2016 and 2018. His research interests are antenna design and electromagnetic metamaterial.

Jiangfeng HAN received the B.S. and M.S. degree from the Information and Navigation Institute, Air Force Engineering University of CPLA, China, in 2012 and 2014, respectively. His research interest is in electromagnetic metamaterials and their antenna applications.

Huanhuan YANG has received the M. Eng. degree in Electronic Science and Technology from the Air Force Engineering University, Xi' an China, in 2012. His research activities focus in polarized reconfigurable antenna. He has authored and coauthored more than 30 scientific papers in major journals and international conferences.

Jianghao TIAN was born in Shaanxi province, China. He received his B.S. degree from the Nanjing University of Posts and Telecommunications in 2017. He currently works towards his M.S. degree. In his research, he specializes in metamaterial and RCS reduction techniques. 